\title{
Treatment of Stifle Joint Luxation in a Cat Using a Temporary Trans-Articular Locking Plate
}

\author{
Chadi Eid $^{1}$ Filippo Lusetti ${ }^{1}$ (1) Andrea Bonardi $^{1}$ Filippo Maria Martini ${ }^{1,2}$ \\ 1 Ortovet stp SRL, Fidenza, Parma, Italy \\ ${ }^{2}$ Department of Veterinary Medical Sciences, University of Parma, \\ Parma, Italy \\ Address for correspondence Chadi Eid, DVM, PhD, Ortovet stp SRL, \\ Piazza Alessandrini, 2-43036 Fidenza (PR), Italy \\ (e-mail: Chadi.eid@ortovet.org).
}

VCOT Open 2021;4:e20-e23.

\begin{abstract}
Keywords

- cat

- stifle

- luxation

- trans-articular plating

The aim of this study was to report the surgical treatment of stifle joint luxation through temporary immobilization of the joint with locking plates in a cat. Stifle joint was immobilized using a temporary trans-articular locking plate applied medially. Return to function was assessed by physical and radiographic follow-up examinations. The cat had good return to function despite a reduction in range of motion. Moderate chronic gonarthritis developed, as seen radiographically. Under the limitation of this single case, temporary trans-articular plating may be considered as useful method for the treatment of long-standing, severely displaced stifle joint luxations in cats.
\end{abstract}

\section{Introduction}

Joint injuries in cats are often secondary to severe trauma such as road accidents and falls from height. Joint luxations occur if several of the joint stabilizers are disrupted. Traumatic stifle joint luxation is an uncommon condition in dogs and cats. The latter appear to have a higher incidence of stifle joint luxation than dogs. ${ }^{1}$

Several treatment options for stifle joint luxations have been reported in the literature, such as intra- ${ }^{2}$ and extracapsular stabilization with or without ${ }^{1}$ trans-articular external fixation, trans-articular pinning with ${ }^{3}$ or without external coaptation, ${ }^{4}$ and arthrodesis. ${ }^{1,5}$

The application of temporary trans-articular locking plates for the treatment of a medial shoulder joint luxation in a dog, and intertarsal and tarsometatarsal instabilities in dogs and cats has been previously reported., ${ }^{6,7}$ To our knowledge temporary trans-articular plating for the treatment of stifle joint luxation has not been previously described. The purpose of this case report is to describe the use and outcome of temporary trans-articular plating for the treatment of a severely dislocated stifle joint luxation in a cat.

received

April 30, 2020

accepted after revision

January 2, 2021
DOI https://doi.org/

$10.1055 / \mathrm{s}-0041-1723939$.

ISSN 2625-2325.

\section{Case Description}

A 6-year-old, male, mixed-breed cat, $6 \mathrm{~kg}$ with a body condition score of 7 out of 9 , was presented for evaluation 35 days after falling from height. The cat was non-weight bearing on the left hind limb with marked instability and swelling of the left stifle. Preoperative radiographic findings were consistent with multiligamentous stifle injury and complete joint luxation.

The cat was placed under general anaesthesia and positioned in dorsal recumbency for surgery. Approach to the medial collateral ligament was performed. ${ }^{8}$ Considering chronic luxation changes, we assumed that stifle joint exploration would not have been beneficial, and therefore arthrotomy was not performed. The stifle joint was manually reduced and flexion was maintained at an angle of 100 to 110 degrees. A 12-hole 2.4 PAX (Securos Surgical AmerisourceBergen, Fiskdale, Massachusetts, United States) reconstruction plate was contoured and applied across the medial aspect of the stifle joint in a similar fashion as reported for stifle arthrodesis ${ }^{5}$ using three screws on the femur and three on the tibia. The incision was closed routinely. Postoperative radiographs ( - Fig. 1) showed good reduction with slight

\section{(c) 2021. The Author(s).}

This is an open access article published by Thieme under the terms of the Creative Commons Attribution License, permitting unrestricted use, distribution, and reproduction so long as the original work is properly cited. (https://creativecommons.org/licenses/by/4.0/)

Georg Thieme Verlag KG, Rüdigerstraße 14, 70469 Stuttgart, Germany 

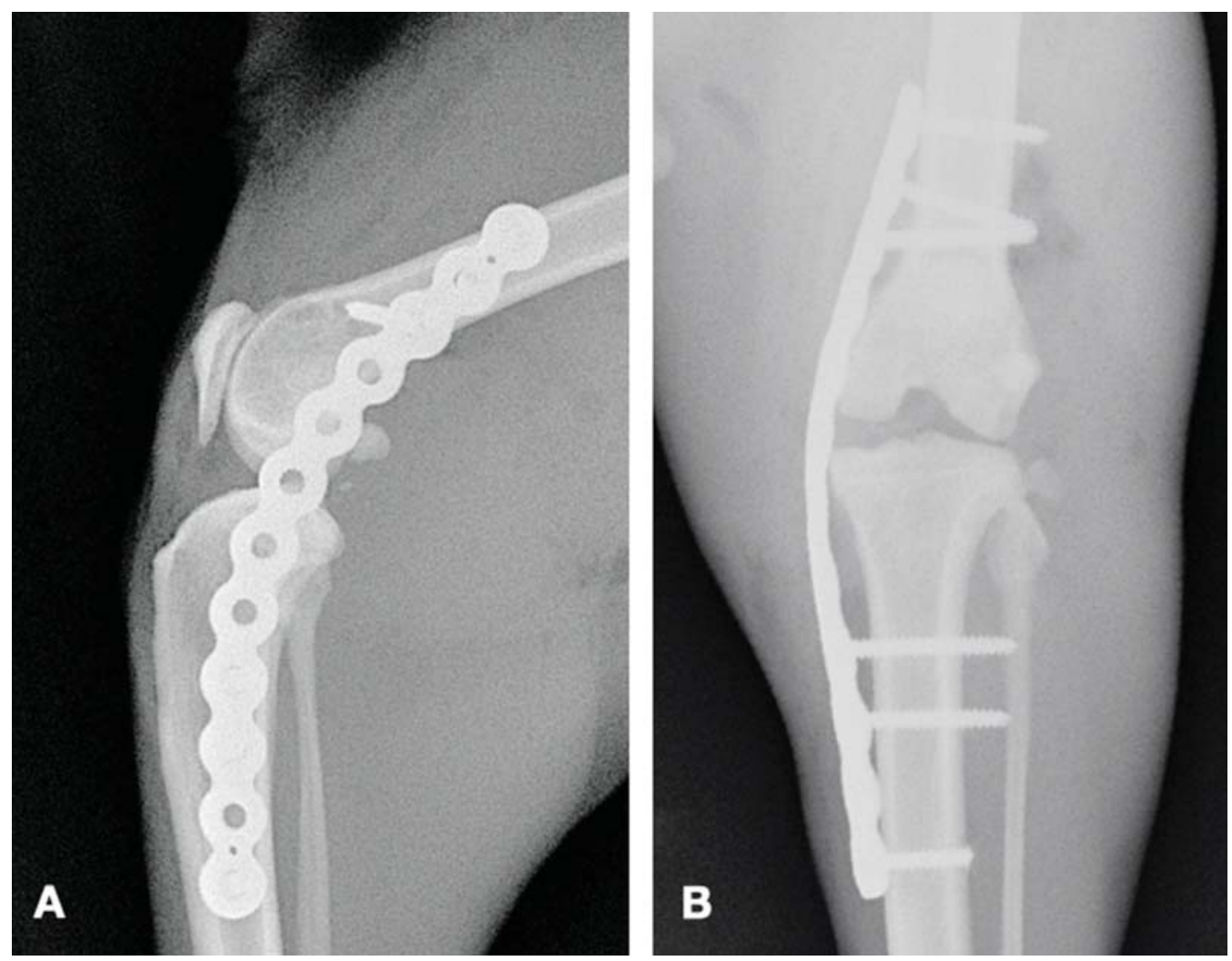

Fig. 1 Postoperative mediolateral (A) and caudocranial (B) views showing good stifle joint reduction and trans-articular PAX plate position.

caudal subluxation of the tibia with some valgus and fixed at an extension angle of 105 degrees. The patient was discharged with instructions given for activity restriction. Meloxicam $(0.1 \mathrm{mg} / \mathrm{kg}$, per os [PO], once daily) for 15 days, cefadroxil ( $20 \mathrm{mg} / \mathrm{kg}$, PO, twice daily) for 7 days and tramadol ( $2 \mathrm{mg} / \mathrm{kg}, \mathrm{PO}$, thrice daily) for 3 days were prescribed.

Follow-up examination performed 2 weeks postoperatively revealed mild left pelvic limb lameness and a slightly circumducting gait without evidence of pain upon palpation.

The implant was removed 8 weeks postoperatively. This required a limited medial approach to the distal femur and proximal tibia of $2 \mathrm{~cm}$ and $3 \mathrm{~cm}$ length, respectively. Extension and flexion were limited to respectively 150 and 90 degrees ( 60 degrees range of motion [ROM]).

The cat improved and had no evidence of lameness on visual gait assessment 1 week after implant removal. No pain was detected. Radiographs taken 9 months postoperatively (-Fig. 2) revealed moderate osteoarthritis. At this time, extension was found normal (155 degrees) while flexion was significantly reduced (to 60 degrees). Further follow-up examinations were not performed since the owner reported that the cat had regained full activity.

\section{Discussion}

In cats, stifle joint luxation is uncommon. ${ }^{1}$ This condition is caused by severe elongation, disruption or avulsion of some or all joint stabilizers. ${ }^{2,4}$ The reconstruction of each individual ligament has been recommended when surgical treatment is indicated. ${ }^{2}$ Frequently these soft tissues are friable and unsuit- able for primary repair. ${ }^{3}$ Thus, repair should be attempted by ligament augmentation and external coaptation or transarticular skeletal fixation. ${ }^{1,4,6,9}$ Although stifle disruption has been successfully managed with trans-articular pinning and/or external coaptation, pin loosening, migration, bending and articular cartilage damage have been reported. However, complications associated with trans-articular pinning seem more likely to be due to inadequate external stabilization. ${ }^{3,4}$ In our case, none of these techniques could be applied because of the difficulties to manage postoperative care in this cat. Therefore, we opted to use a technique hitherto only described in dogs: the use of temporary locking plates had been described to treat a luxated shoulder joint in a dog. ${ }^{6}$ However, the need for an additional surgery for implant removal has to be accepted as disadvantage of internal fixation.

Given chronicity, no attempt was made to repair torn ligaments and the stifle was reduced and temporarily transfixed without arthrotomy. The recommended angle for arthrodesis of 110 degrees was slightly exceeded. In retrospect, a more flexed angle may have been advantageous but could have predisposed to implant failure or peri-implant bone fracture. ${ }^{5}$ Biomechanically, we speculated that the construct would have behaved similarly to a bridged large gap fracture. We thought to reduce the risk for implant failure by bridging the joint with a long plate that allows better stress distribution through large working length and less screws. ${ }^{10}$ In addition, the medial position of the plate allows increasing the moment of inertia of the construct and better distribution of the stress across the bone. ${ }^{5}$ The possibility of allowing a gap between plate and surface of the bones, that is, without the need for 

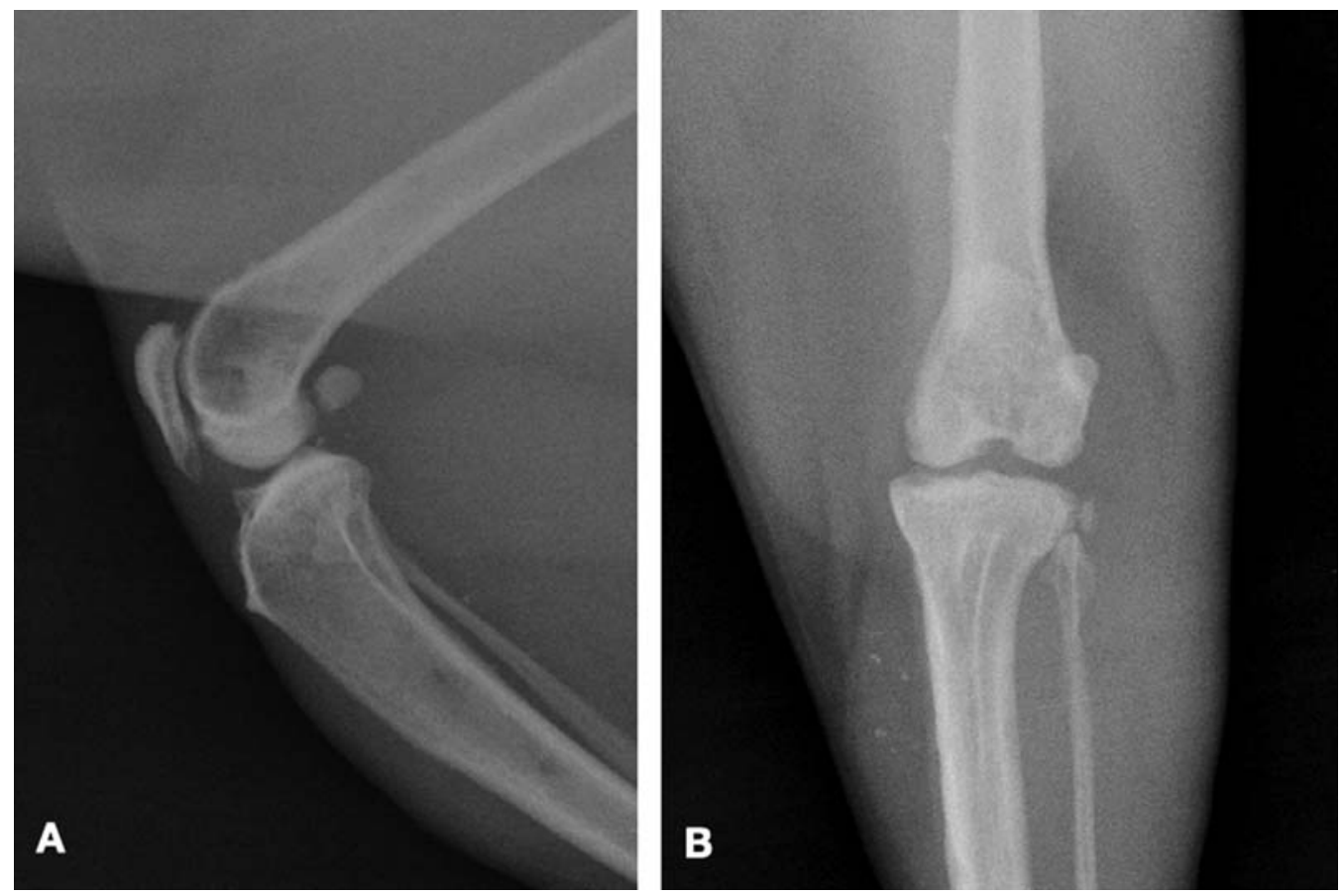

Fig. 2 Mediolateral (A) and caudocranial (B) radiographs taken 9 months postoperatively (7 months after implant removal) revealing moderate osteoarthritis of the stifle joint.

precise contouring of the locking plate, ${ }^{10}$ was an advantage in our case. The mechanical coupling formed between the plate and the locking screws generates an internal fixator that does not require friction between the plate and the bone, thereby preserving blood supply ${ }^{10}$ and allowing fibrous healing of soft tissues in the bone/plate interface. ${ }^{6,10}$

The aim of joint immobilization is to allow adequate fibrous healing of the periarticular soft tissues to achieve sufficient joint stability. Optimal duration of joint immobilization to allow soft tissue repair without causing excessive joint stiffness and irreversible degenerative changes is currently not described. ${ }^{6}$ However, long-term joint immobilization induces substantial changes on articular structures, and some of its effects may be irreversible; it causes reduction in ROM, increase of synovial fluid production and induces cartilage stiffness and loss of cartilage matrix proteoglycan. ${ }^{3,9}$ In our case, stifle joint ROM had improved 9 months after implant removal, but remained limited to 95 degrees. This ROM decrease could be the consequence of the untreated meniscal lesions but, in our opinion, is more likely to be due to chronicity, prolonged immobilization (8 weeks) and periarticular fibrosis. Regardless of the surgical method used, ROM decrease has been reported after stifle joint luxation. ${ }^{1,4} \mathrm{We}$ hypothesized that longer immobilization could be needed in chronic cases. Thus, the recommended immobilization time of 5 to 7 weeks ${ }^{4}$ for the stifle joint has been slightly exceeded in our case. Nevertheless, ROM reduction has also been reported with shorter immobilization times ${ }^{4}$ and, albeit to a lesser degree, even with the use of hinged external fixation. ${ }^{9}$

The development of osteoarthrosis was not surprising; mild to moderate degenerative joint changes after stifle joint luxation were noted also in other reports and it is known that these changes increase with longer immobilization periods. ${ }^{1,2,4}$ In addition, implant removal represents a sudden remobilization of the joint that may damage the structural integrity of the already altered articular cartilage. ${ }^{9}$

In conclusion, the treatment of this cat was considered a salvage approach. Despite the substantial reduction in ROM the cat regained full activity and since the alternative would have been arthrodesis or more likely amputation, we consider satisfactory the clinical outcome. This technique is not meant to replace primary repair of torn ligaments and/or dynamic temporary fixation, but, in selected cases, temporary locking plate fixation could be a valid option for the treatment of longstanding, massive stifle joint luxation as an alternative to external fixation and trans-articular pinning. Further investigations and more clinical cases are needed to establish whether trans-articular plating ensures long-term joint stability with minor collateral damage. Until the optimal duration of joint immobilization by temporary plating is established, it would be preferable to adopt the minimum conventional immobilization time of 5 weeks even in the case of chronic luxations.

\section{Conflict of Interest}

A.B. reports grants from Intrauma, outside the submitted work. C.E. reports grants from Intrauma, outside the submitted work. F.L. reports grants from Intrauma, outside the submitted work. F.M.M. reports grants from Intrauma and from Securos, outside the submitted work.

\section{References}

1 Bruce WJ. Stifle joint luxation in the cat: treatment using transarticular external skeletal fixation. J Small Anim Pract 1999;40 (10):482-488

2 Hulse DA, Shires PK. Multiple ligament injury of the stifle joint in the dog. J Am Anim Hosp Assoc 1986;22:105-110 
3 Connery NA, Rackard S. The surgical treatment of traumatic stifle disruption in a cat. Vet Comp Orthop Traumatol 2000;13:208-211

4 Welches CD, Scavelli TD. Transarticular pinning to repair luxation of the stifle joint in dogs and cats: a retrospective study of 10 cases. J Am Anim Hosp Assoc 1990;26:207-214

5 Belch A, Fitzpatrick N, Farrell M. Stifle arthrodesis in two cats. Vet Comp Orthop Traumatol 2012;25(05):421-426

6 Post C, Guerrero T, Voss K, Montavon PM. Temporary transarticular stabilization with a locking plate for medial shoulder luxation in a dog. Vet Comp Orthop Traumatol 2008;21(02):166-170

7 Voss K, Keller M, Montavon PM. Internal splinting of dorsal intertarsal and tarsometatarsal instabilities in dogs and cats with the
ComPact Unilock 2.0/2.4 (TM) system. Vet Comp Orthop Traumatol 2004;17:125-130

8 Johnson KA. Piermattei's Atlas of Surgical Approaches to the Bones and Joints of the Dog and Cat. 5th edition Philadelphia: Saunders; 2015

9 Jaeger GH, Wosar MA, Marcellin-Little DJ, Lascelles BD. Use of hinged transarticular external fixation for adjunctive joint stabilization in dogs and cats: 14 cases (1999-2003). J Am Vet Med Assoc 2005;227(04):586-591

10 Gautier E, Sommer C. Guidelines for the clinical application of the LCP. Injury 2003;34(Suppl 2):B63-B76 